\title{
Saphenous Vein Valves Assessment Utilizing Upright CT. -Potentially Improve the Graft Assessment for Bypass Surgery-
}

\author{
Takehiro Nakahara \\ Keio University \\ Minoru Yamada \\ Keio University \\ Yoichi Yokoyama \\ Keio University \\ Yoshitake Yamada \\ Keio University \\ Keiichi Narita \\ Keio University \\ Nobuaki Imanishi \\ Keio University \\ Masataka Yamazaki \\ Keio University \\ Hideyuki Shimizu \\ Keio University \\ Jagat Narula \\ Icahn School of Medicine at Mount Sinai \\ Masahiro Jinzaki ( $\boldsymbol{\nabla}$ jinzaki@rad.med.keio.ac.jp) \\ Keio University
}

\section{Research Article}

Keywords: Upright CT, saphenous vein, valve, tributaries, vessel size

Posted Date: January 21st, 2021

DOI: https://doi.org/10.21203/rs.3.rs-144873/v1

License: (a) (i) This work is licensed under a Creative Commons Attribution 4.0 International License.

Read Full License 


\section{Abstract}

The saphenous veins (SV) are frequently employed as bypass grafts. The SV graft failure is predominantly seen at the valve site. Avoiding valves during vein harvest would help reduce graft failure. We endeavored to detect SV valves, tributaries, and vessel size employing upright CT for the raw cadaver venous samples and in healthy volunteers.

Five cadaver legs were scanned. The anatomical analysis showed 3.0 (IQR: 2.0-3.0) valves and 13.50 (IQR: 10.00-16.25) tributaries. The upright CT completely detected, compared to 2.0 (IQR: 1.5-2.5, $\mathrm{p}=0.06$ ) valves and 9.5 (IQR: 7.5-13.0, $\mathrm{p}=0.13$ ) tributaries by supine CT.

From a total 190 volunteers, 138 (men:75, women:63) were included. The number of valves from the SF junction to $35 \mathrm{~cm}$ were significantly higher in upright CT than in supine CT bilaterally [upright vs. supine, Right: 4 (IQR: 3-5) vs. 2 (IQR:1-2), p<0.0001, Left: 4 (IQR: 3-5) vs. 2 (IQR: 1-2), p <0.0001]. The number of tributaries and vessel area was also higher for upright compared with supine CT.

Upright CT enable detection of SV valves, tributaries, and vessel size non-invasively. Although not tested here, it is expected that upright CT might potentially improve the graft assessment for bypass surgery.

\section{Introduction}

For decades, the saphenous vein (SV) has served as the most reliable bypass conduits for coronary and peripheral artery revascularization. Over 250,000 coronary artery bypass grafts and over 80,000 lower extremity vein grafts are performed annually in the United States alone ${ }^{1}$. The average rate of CABG has been reported to be 44 per 100,000 individuals ${ }^{2}$, and almost $80 \%$ of bypass grafts include saphenous veins (SV) ${ }^{2,3}$. However, the SV graft failure during the follow-up is not uncommon ${ }^{3,4}$ wherein most stenotic lesions are focal and often occur either at the valve sites or anastomotic regions ${ }^{5-7}$. Therefore, it has been proposed that not including the venous samples with valve sites may reduce the SV graft failure.

The valves of venous systems with venous muscle pumps allow the blood to return to the heart against gravity, and generally demonstrate expansion at the valve site during upright position ${ }^{8}$. We have developed an upright CT (Figure.1 A, B) to negate the postural effect of gravity and render the clinical imaging as close to physiology as possible ${ }^{9-11}$. Employing the upright $C T$, we aimed to detect SV valves, tributaries and venous area compared with standard supine CT imaging.

\section{Materials And Methods}

Cadaver study. Cadaveric specimens were obtained from the Willed Body Program (Department of Anatomy, Keio University School of Medicine) and were investigated in accordance with institutional regulations. Lower extremities, with no scars and no signs of vascular disease, were obtained from raw cadavers. Lower extremities were disarticulated at the hip joint and the external iliac artery, external iliac 
vein and the surrounding soft tissue remained attached to the specimens. The distal position of saphenous vein was cannulated with 22-Gauze catheter at above ankle level and phosphate buffered saline (PBS) with $10 \%$ polyethylene glycol ${ }^{12}$ was infused during the scan to reproduce the venous reflex. The CT images were acquired as ex-vivo imaging of the disarticulated leg. Thereafter, the leg specimens were fixed in formaldehyde and the saphenous vein was dissected and made a long single incision along the venous path from the cannulation point to the saphenous-femoral junction (SF junction) (Supplemental Figure.1). The SV valves and tributaries positions were anatomically identified as the reference standard for CT images.

Volunteer study. The volunteer prospective study was approved by the institutional review board (Keio University Independent Ethics Committee) (Clinical Trail number: UMIN000026586), and written informed consent was obtained from all participants. Healthy volunteers were recruited from a volunteer recruitment company from July 2017 to March 2019; volunteers of over 30 years of age were requested so that they could understand the purpose of the study. Individuals with a history of hypertension, dyslipidemia, diabetes, smoking, or those who had previously undergone cardiac surgery or were currently receiving treatment were excluded.

Image acquisition. A conventional 320-detector row CT (Aquilion ONE, Canon Medical Systems Corporation, Otawara, Japan) was prospectively performed for all volunteers in the supine position. In addition, and upright CT (prototype TSX-401R, Canon Medical Systems Corporation, Otawara, Japan) ${ }^{9}$ was performed in standing position immediately after the conventional procedure. The upright and conventional supine CT scanners were next to each other, and the two examinations were consecutively performed. The upright CT system is characterized by up-and-down movements of a transverse 320 rowdetector gantry (isotropic $0.5 \mathrm{~mm}$ in detector size), with a bore size of $780 \mathrm{~mm}$, a gantry rotation speed of $0.275 \mathrm{~s}$, maximum vertical speed of $100 \mathrm{~mm} / \mathrm{s}$, and a 1200 view at optimal performance. Scanning was performed at $100 \mathrm{kVp}$ and a gantry rotation speed of $0.5 \mathrm{~s}$ in the helical scan mode (80-row detector), with a noise index of 24 and helical pitch of 0.8 for the body trunk from the level of superior margin of the external acoustic meatus to the lowest position of the upright CT ( $37 \mathrm{~cm}$ height).

For cadaver study, cadaver legs were placed in plastic moulds and scanned whole samples in both supine and upright CT scanners. The plastic box containing the sample was fixed on a pedestal $(42 \mathrm{~cm}$ height) and tube current for scanning was set as approximately $600 \mathrm{~mA}$ to compensate the noise from the fixtures.

Image reconstruction was performed using Adaptive Iterative Dose Reduction 3D (Canon Medical Systems Corporation, Otawara, Japan), which could reduce the radiation dose.

Image analysis. CT images were transferred to an off-line workstation (SYNAPSE VINCENT, FujiFilm, Tokyo, Japan) and multiplanar reconstruction (MPR) images were developed from the SF junction of saphenous vein both for cadaveric images and clinical images. The position and number of valves were counted visually. Simultaneously, the vessel area in cross-sectional images was measured to calculate 
dilation ratio (vessel area at valve /vessel area at reference segments). The reference segments were set at the average of the vessel area of proximal and distal position of the valves. The dilation ratio was derived in the cadaveric study and applied for the volunteer study to study the valves. The number of tributaries were counted visually. The vessel area was measured of the cross-section image on the $15 \mathrm{~cm}$ from the SF junction were measured. For cadaveric study, two observers analyzed the data with consensus reading. For volunteer study, all measurements were performed in a blinded and randomized manner.

Statistical analysis. Intra-observer and inter-observer variability for the assessment of valves, tributaries, and cross section measurements were examined using Bland-Altman analysis and Pearson's correlation. Data were presented as median (interquartile range [IQR]; i.e., 25th to 75th percentile, or Q1, Q3). Continuous data were compared using Wilcoxon rank sum test between the 2 groups. The Spearman Rank Correction coefficient test was used for the assessment of linear correlation of 2 parameters. A 2sided $p<0.05$ was considered statistically significant. All analyses were performed using SAS software, version 9.4. (SAS Institute Inc., Cary, North Carolina).

\section{Results}

Cadaver study. Five legs were dismembered from cadavers (age range,78-93 years, mean $88.4 \pm 6.0$ years, 4 left and 1 right leg, 4 female and 1 male). On visual assessment 3.0 (IQR 2.0-3.0) valves and 13.50 (IQR 10.00-16.25) tributaries were observed in SV (Figure. 1 C-E).

The upright CT images accurately identified the valves and tributaries; the identification was difficult by the conventional supine CT. The upright CT image showed 3.0 (IQR 2.0-3.0) valves and 13.50 (IQR 10.0016.25) tributaries, while supine CT image showed 2.0 (IQR 1.5-2.5, $p=0.06$ ) valves and 9.5 (IQR 7.5- 13.0, $p=0.13$ ) tributaries; given a small sample size the difference however was not statistically significant. The expansive venous diameter at the valve site (referred to as dilation ratio) in the upright CT images was 1.56 (IQR 1.44-1.83, range 1.20-4.24), and the minimum dilation ratio of "1.20" was used to confirm the valve site in the volunteer images.

Volunteer study. Of 190 volunteers, 38 who received the scan with $120 \mathrm{KV}$ were excluded; another 14 volunteers were excluded where complete $C T$ data from the SF junction to $35 \mathrm{~cm}$ could not be obtained due to the short stature. We report the CT characteristics of SV from 138 volunteers (men 75, women 63) who completed the study (Table.1).

Firstly, 40 CT leg images from 10 consecutive volunteers, both sides and both supine and upright positions, were analyzed by two observers; high intra-/inter- observer agreement was noted (Supplemental Figure 2, 3 and 4). The upright images allowed identification of the valves and tributaries (Figure. 2). It was difficult to characterize valves and tributaries in supine CT images similar to cadaveric study. 
The number of valves were significantly higher in upright CT than in supine CT on both sides [upright right side: 4 (IQR: 3-5) vs. supine right side: 2 (IQR: 1-2), p< 0.0001, upright left side: 4 (IQR: 3-5) vs. supine left side: 2 (IQR: 1-2), $p<0.0001$ ] (Figure.3A and B). The dilation ratio at the valve site was 1.85 (IQR 1.572.25). The frequency of valve distribution was maximum between 0 to $5 \mathrm{~cm}$ from the $S F$ junction, followed by 5 to $10 \mathrm{~cm}$ from the SF junction (Figure.3C).

The number of tributaries was also higher in the upright CT compared with the supine CT [upright right: 12 (IQR: 10-15) vs. supine right:11 (IQR: 9-13), p< 0.0001; upright left: 12 (IQR: 9-14) vs. supine left:11 (IQR: 9-13), $\mathrm{p}<0.0001$ ] (Figure.4A).

The area of cross section image at $15 \mathrm{~cm}$ from the SF junction was larger in the upright CT [upright right side: 14 (IQR: 11-17) $\mathrm{mm}^{2}$ vs. supine right: 12 (IQR: 9-16) $\mathrm{mm}^{2}, \mathrm{p}<0.0001$; upright left: 14 (IQR: 11-18) $\mathrm{mm}^{2}$ vs. supine left:12 (IQR: 9-15) $\mathrm{mm}^{2}, \mathrm{p}<0.0001$ ] (Figure.4B).

\section{Discussion}

This study showed that the SV valves were clearly visualized in the standing position utilizing upright CT both in cadaveric legs and in-vivo study of healthy volunteers. In the cadaver study, SV valves and tributaries identified by upright CT were confirmed by anatomical analysis. The minimum dilation ratio of " 1.20 " was found to be the cut-off point to define the level of the valve to confirm the valve site in the invivo study. In the volunteer study, upright CT detected larger number of valves and tributaries and larger size of vessel area comparison with supine CT. We propose that upright CT could have an advantage for SV characterization prior to bypass surgeries.

The SV grafts have surgeries for long due to their easy availability, they are more resistant to iatrogenic injury, less susceptible to vasospasm ${ }^{13}$ and provide longer length compared with other kind of grafts. The SV grafts have been most used for non-LAD coronary territories worldwide. It has major disadvantage of high graft failure rate ${ }^{2,3}$; approximately $40-50 \%$ grafts occlude at 10 years after the CABG surgery, of which $10-25 \%$ are occluded within first year post-operatively $4,14,15$. In comparison, the occlusion rate of other grafts is lower, for instance the radial artery shows $17-37 \%$ failure rate ${ }^{14,16}$. One of the several causes of SV failure is the endothelium dysfunction during the harvest and new harvesting techniques including bridge, no-touch, endoscopic resection help reduce the dysfunction and the graft failure. The other underlying cause has been reported as the presence of venous vales. Although the mechanisms of primary stenosis of coronary artery bypass graft and peripheral artery bypass graft have been suggested to be different, they share thrombosis as the basis of luminal loss ${ }^{17}$. In SV grafts, valve sites frequently gather clots ${ }^{18}$, which cause valve dysfunction, scarring and develop post-thrombotic syndrome ${ }^{17}$; the response of local smooth muscle cells to injury is found to be accelerated in-vitro ${ }^{19}$. The clinical studies have confirmed that the valve sites frequently cause stenotic lesions when SV graft reoperations are performed ${ }^{5-7,20-22}$. We assumed that knowing $S V$ valve position and avoiding the valve sites during harvesting may offer another approach to reduce the SV graft failure. 
To identify the best portion of the SV as a conduit for bypass surgery, the number and position of valves in SV were reviewed. A Brazilian study characterized 60 veins from 30 adult cadavers and reported that the average number of the valves from the medial epicondyle of the femur to the saphenous hiatus were 4.77 and 4.87 on the left and right side, respectively ${ }^{23}$. Similarly a Japanese study of 26 SV from 20 adult cadavers found 111 valves (average: 4.27 ) between the SF junction and the upper patellar margin compared to 63 (average: 2.42) valves between the upper patellar margin and the medial malleolus 24 ; they also reported that the valves most valves were observed within $10 \mathrm{~cm}$ from the SF junction and between $35-45 \mathrm{~cm}$ from the SF junction. Because we identified 4 (IQR: 3-5) valves from between $0 \mathrm{~cm}$ to $35 \mathrm{~cm}$ from the SF junction and the distribution of valves was mainly observed between 0 to $10 \mathrm{~cm}$, the results of our volunteer study were comparable with these autopsy studies; however, the data beyond 35 $\mathrm{cm}$ from the SF junction was not available in this study. Therefore, the upright CT demonstrated an accurate characterization of SV valves not easily possible by the conventional computed tomography.

Another merit of the upright CT was the noninvasive identification of tributaries and vessel area. Vessel size is an important factor to predict graft failure and a luminal diameter of over $2.0 \mathrm{~mm}$ is preferable for SV graft patency and longevity ${ }^{3,14,25}$. New harvesting techniques less touch SV may reduce the damage compared with conventional open harvesting technique, however; it is difficult to evaluate the valve sites and the vessel size in the whole SV. In addition, it is important to be able to ligate all side tributaries ${ }^{26}$. The upright CT allowed to locate valves, identify tributaries and accurately define vessel size noninvasively and should assist the strategy for harvesting.

Recently upright (or weight-bearing) CT ${ }^{27}$ and MRI ${ }^{28}$ have been proposed to evaluate the effect of gravity. However, the previous upright CT was equipped with a cone beam CT and scan range was limited. The upright MRI has lower spatial resolution compared with $\mathrm{CT}^{29}$. Compared to these machines, the upright CT used in the current study carries a wide-scan range with quick motion and high spatial resolution, similar to a high-end supine $\mathrm{CT}$ at standing position. It has an ability to evaluate blood distribution of whole body ${ }^{9}$, and may contribute to the development of the Phlebology, which has room for the development compared with the Arteriology.

Limitations of the study. Although a novel attempt, this study has several limitations. First, since the upright CT is a prototype machine, this is a single center study. Second, the protocol of this study was designed to obtain body-trunk images and the scan range of this prototype machine could not detect beyond $37 \mathrm{~cm}$ height, not allowing the images of entire saphenous vein. However, we could acquire the whole SV with pedestal with $42 \mathrm{~cm}$ height, which we utilized for the evaluation of ankles ${ }^{30}$. Third, the valve insertion sites were identified by defining venous (expansive) dilation ratio compared to the lumen and a cut-off point of 1.20 was based on the anatomic observation in the cadaveric limb. An application of workstation is being developed to enable detecting the valve automatically in future clinical studies. Fourth, we could not identify the valve leaflet directly, because the upright CT scans were performed without contrast medium and the limit of the spatial resolution of CT is 0.4 to $0.6 \mathrm{~mm}$. The invasive OCT with 15 micro-meter spatial resolution ${ }^{29}$ have allowed to recognize the culprit valve lesions after CABG 
31. However, it is difficult to identify the valves of SV in supine CT even with contrast materials. Thus, this approach will be practically useful to detect valves positions.

\section{Conclusions}

Upright CT enables detection of SV valve sites, tributaries, and vessel size noninvasively. It may potentially assist the strategies for SV harvesting to improve patency of bypass-grafts.

\section{Declarations}

\section{Acknowledgments}

This study was supported by Canon Medical Systems, Japan Society for the Promotion of Science (JSPS KAKENHI: grant number JP17H04266), Uehara Memorial Foundation, and Canon Medical Systems (Otawara, Japan) to JM.

\section{Author contributions}

T.N. made contribution to the conception and design of the study, acquisition, analysis and interpretation of cadaver study data and volunteer study data, and drafting the article. Mi.Y., Y.Yo., made contribution to the acquisition of cadaver study data and volunteer study data, and revising the manuscript critically for important intellectual content. Y.Ya. and K.N. made contribution to the acquisition of volunteer study data and revising the manuscript critically for important intellectual content. N.I., Ma.Y. H.S. and J.N. made contribution to revising the manuscript. M.J. made contribution to the conception and design of the study and revising the manuscript.

\section{Conflict of Interest}

This study was supported by Japan Society for the Promotion of Science (JSPS KAKENHI: grant number JP17H04266), Uehara Memorial Foundation, and Canon Medical Systems (Otawara, Japan). Masahiro Jinzaki has received a grant from Canon Medical Systems. Canon Medical Systems has loaned the upright computed tomography machine to Keio University. However, Canon Medical Systems is not involved in the design and conduct of the study; in the collection, analysis, and interpretation of the data; and in the preparation, review, or approval of the manuscript. All other authors have no relationships with industry or other entities.

\section{Data availability}

The datasets generated during and/or analysed during the current study are available from the corresponding author on reasonable request.

\section{References}


1. Bhasin, M. et al. Temporal network based analysis of cell specific vein graft transcriptome defines key pathways and hub genes in implantation injury. PLoS One 7, e39123, doi:10.1371/journal.pone.0039123 (2012).

2. Head, S. J., Milojevic, M., Taggart, D. P. \& Puskas, J. D. Current Practice of State-of-the-Art Surgical Coronary Revascularization. Circulation 136, 1331-1345, doi:10.1161/circulationaha.116.022572 (2017).

3. Caliskan, E. et al. Saphenous vein grafts in contemporary coronary artery bypass graft surgery. Nat Rev Cardiol, doi:10.1038/s41569-019-0249-3 (2019).

4. Fitzgibbon, G. M. et al. Coronary bypass graft fate and patient outcome: angiographic follow-up of 5,065 grafts related to survival and reoperation in 1,388 patients during 25 years. J Am Coll Cardiol 28, 616-626, doi:10.1016/0735-1097(96)00206-9 (1996).

5. Bandyk, D. F., Schmitt, D. D., Seabrook, G. R., Adams, M. B. \& Towne, J. B. Monitoring functional patency of in situ saphenous vein bypasses: the impact of a surveillance protocol and elective revision. J Vasc Surg 9, 286-296 (1989).

6. Mills, J. L., Fujitani, R. M. \& Taylor, S. M. The characteristics and anatomic distribution of lesions that cause reversed vein graft failure: a five-year prospective study. J Vasc Surg 17, 195-204; discussion 204-196, doi:10.1067/mva.1993.42796 (1993).

7. Davies, M. G. \& Hagen, P. O. Pathophysiology of vein graft failure: a review. Eur J Vasc Endovasc Surg 9, 7-18, doi:10.1016/s1078-5884(05)80218-7 (1995).

8. Bergan, J. J. et al. Chronic venous disease. N Engl J Med 355, 488-498, doi:10.1056/NEJMra055289 (2006).

9. Jinzaki, M. et al. Development of Upright Computed Tomography With Area Detector for Whole-Body Scans: Phantom Study, Efficacy on Workflow, Effect of Gravity on Human Body, and Potential Clinical Impact. Invest Radio/ 55, 73-83, doi:10.1097/rli.0000000000000603 (2020).

10. Yamada, Y. et al. Differences in Lung and Lobe Volumes between Supine and Standing Positions Scanned with Conventional and Newly Developed 320-Detector-Row Upright CT: Intra-Individual Comparison. Respiration 99, 598-605, doi:10.1159/000507265 (2020).

11. Narita, K. et al. Pelvic floor morphology in the standing position using upright computed tomography: age and sex differences. Int Urogynecol J, doi:10.1007/s00192-020-04335-z (2020).

12. Yamazaki, S. et al. Three-dimensional demonstration of the lymphatic system in the lower extremities with multi-detector-row computed tomography: a study in a cadaver model. Clin Anat 26, 258-266, doi:10.1002/ca.22179 (2013).

13. Parang, P. \& Arora, R. Coronary vein graft disease: pathogenesis and prevention. Can J Cardio/25, e57-62, doi:10.1016/s0828-282x(09)70486-6 (2009).

14. Goldman, S. et al. Long-term patency of saphenous vein and left internal mammary artery grafts after coronary artery bypass surgery: results from a Department of Veterans Affairs Cooperative Study. J Am Coll Cardio/ 44, 2149-2156, doi:10.1016/j.jacc.2004.08.064 (2004). 
15. Hess, C. N. et al. Saphenous vein graft failure after coronary artery bypass surgery: insights from PREVENT IV. Circulation 130, 1445-1451, doi:10.1161/circulationaha.113.008193 (2014).

16. Motwani, J. G. \& Topol, E. J. Aortocoronary saphenous vein graft disease: pathogenesis, predisposition, and prevention. Circulation 97, 916-931, doi:10.1161/01.cir.97.9.916 (1998).

17. ten Cate-Hoek, A. J., Henke, P. K. \& Wakefield, T. W. The post thrombotic syndrome: Ignore it and it will come back to bite you. Blood Rev 30, 131-137, doi:10.1016/j.blre.2015.09.002 (2016).

18. Mackman, N. New insights into the mechanisms of venous thrombosis. J Clin Invest 122, 2331-2336, doi:10.1172/jci60229 (2012).

19. Kikuchi, S. et al. Smooth muscle cells of human veins show an increased response to injury at valve sites. J Vasc Surg 67, 1556-1570.e1559, doi:10.1016/j.jvs.2017.03.447 (2018).

20. Szilagyi, D. E., Elliott, J. P., Hageman, J. H., Smith, R. F. \& Dall'olmo, C. A. Biologic fate of autogenous vein implants as arterial substitutes: clinical, angiographic and histopathologic observations in femoro-popliteal operations for atherosclerosis. Ann Surg 178, 232-246, doi:10.1097/00000658197309000-00002 (1973).

21. Tullis, M. J., Primozich, J. \& Strandness, D. E., Jr. Detection of "functional" valves in reversed saphenous vein bypass grafts: identification with duplex ultrasonography. $J$ Vasc Surg 25, 522-527, doi:10.1016/s0741-5214(97)70263-5 (1997).

22. Vesti, B. R., Primozich, J., Bergelin, R. O. \& Strandness, E., Jr. Follow-up of valves in saphenous vein bypass grafts with duplex ultrasonography. J Vasc Surg 33, 369-374, doi:10.1067/mva.2001.111744 (2001).

23. Portugal, I. B., Ribeiro Ide, L., Sousa-Rodrigues, C. F., Monte-Bispo, R. F. \& Rocha, A. C. Distribution of saphenous vein valves and its practical importance. Rev Bras Cir Cardiovasc 29, 564-568, doi:10.5935/1678-9741.20140038 (2014).

24. Shinohara, H., Morisawa, S., Toshima, M. \& Mizukami, S. Distribution of valves in the great saphenous vein; its clinical implications. Okajimas Folia Anat Jpn 67, 219-221, doi:10.2535/ofaj1936.67.4_219 (1990).

25. Souza, D. S. et al. Improved patency in vein grafts harvested with surrounding tissue: results of a randomized study using three harvesting techniques. Ann Thorac Surg 73, 1189-1195, doi:10.1016/s0003-4975(02)03425-2 (2002).

26. Souza, D. S. et al. Harvesting the saphenous vein with surrounding tissue for CABG provides longterm graft patency comparable to the left internal thoracic artery: results of a randomized longitudinal trial. J Thorac Cardiovasc Surg 132, 373-378, doi:10.1016/j.jtcvs.2006.04.002 (2006).

27. Hirschmann, A., Pfirrmann, C. W., Klammer, G., Espinosa, N. \& Buck, F. M. Upright cone CT of the hindfoot: comparison of the non-weight-bearing with the upright weight-bearing position. Eur Radio/ 24, 553-558, doi:10.1007/s00330-013-3028-2 (2014).

28. Alyas, F., Connell, D. \& Saifuddin, A. Upright positional MRI of the lumbar spine. Clin Radiol 63, 10351048, doi:10.1016/j.crad.2007.11.022 (2008). 
29. Nakahara, T. et al. Coronary Artery Calcification: From Mechanism to Molecular Imaging. JACC Cardiovasc Imaging 10, 582-593, doi:10.1016/j.jcmg.2017.03.005 (2017).

30. Kaneda, K. et al. Three-dimensional kinematic change of hindfoot during full weightbearing in standing: an analysis using upright computed tomography and 3D-3D surface registration. J Orthop Surg Res 14, 355, doi:10.1186/s13018-019-1443-z (2019).

31. Koeda, Y., Itoh, T., Fusazaki, T., Nakamura, M. \& Morino, Y. A unique stenosis in saphenous vein graft visualized by optical coherence tomography. Heart Vessels 29, 278-281, doi:10.1007/s00380-0130362-x (2014).

\section{Tables}

Due to technical limitations, table 1 is only available as a download in the Supplemental Files section.

\section{Figures}


(A)

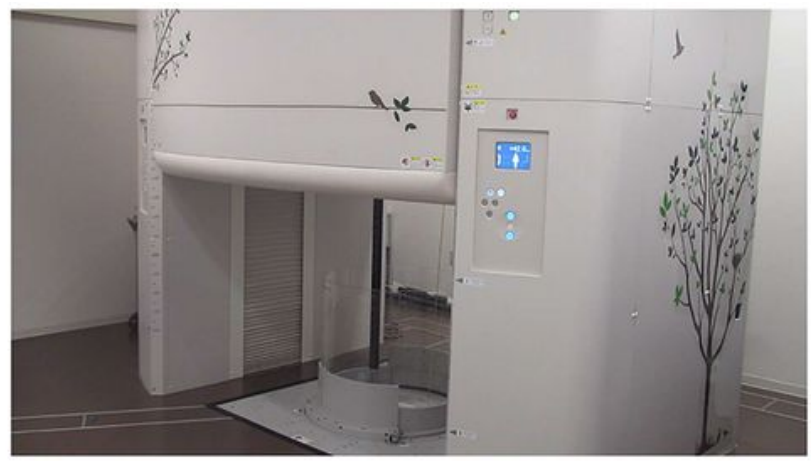

(B)

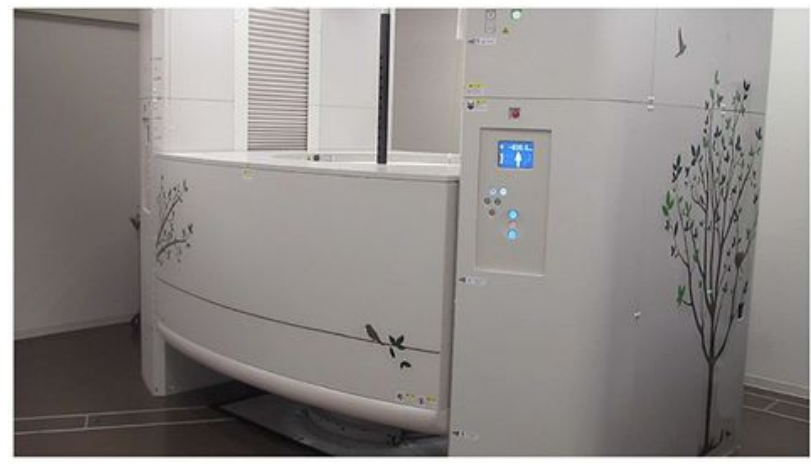

(C)

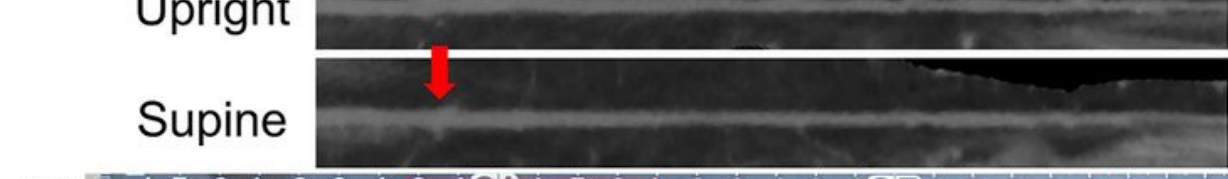

(D)

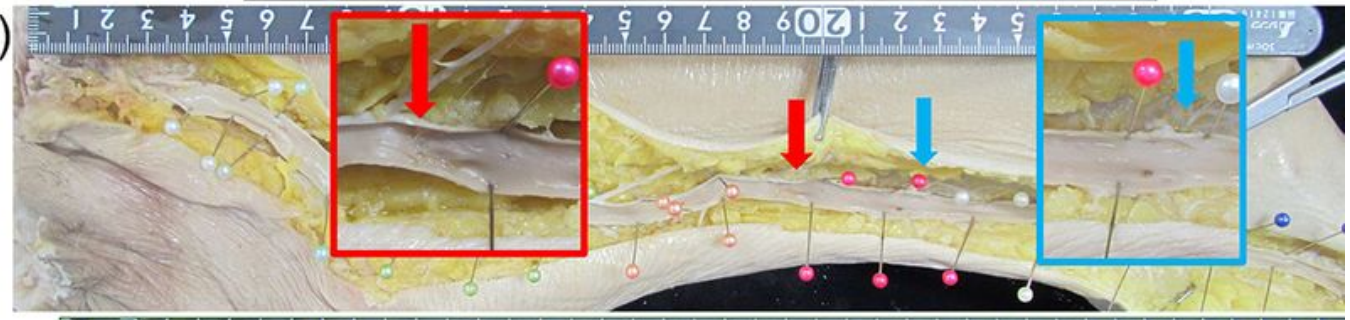

(E)

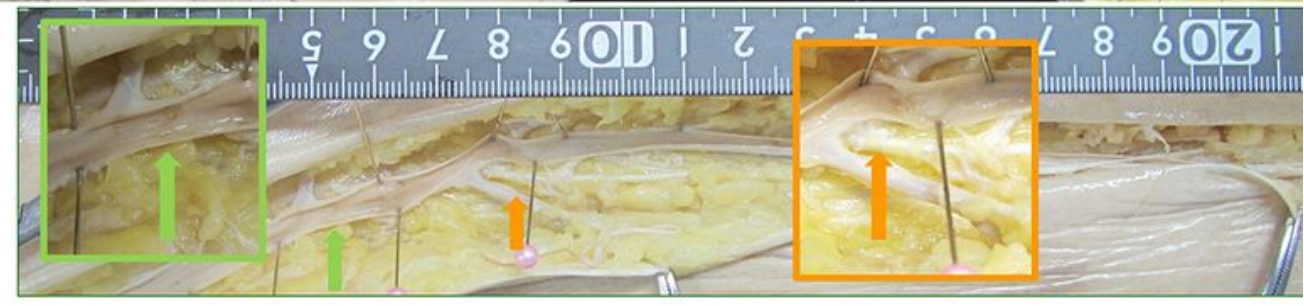

\section{Figure 1}

An overview of upright CT and CT images of raw cadaver lower extremities and anatomical analyses after fixed with formaldehyde. (A) Gantry in the up position. (B) Gantry in the down position. Subjects stand in the center of the gantry and the gantry moves up to down to scan. (C) MPR images of SV based on both upright CT and supine CT. Four valves (arrows) were identified in upright CT, however; some of them had difficulty to identify in supine CT. (D and E) The upper part (D) and lower part (E) of the same 
lower extremity. After image acquisitions, the specimen samples were fixed by formaldehyde and saphenous vein was dissected and made a long single incision along the venous path from the cannulation point to the SF junction. Four valves were identified in anatomical analysis (arrows. the color of the arrows was corresponded in the CT images in panel C) and confirmed the results of upright CT analysis. The tributaries were also analyzed in the same manner.

(A)

Right

Upright

Supine
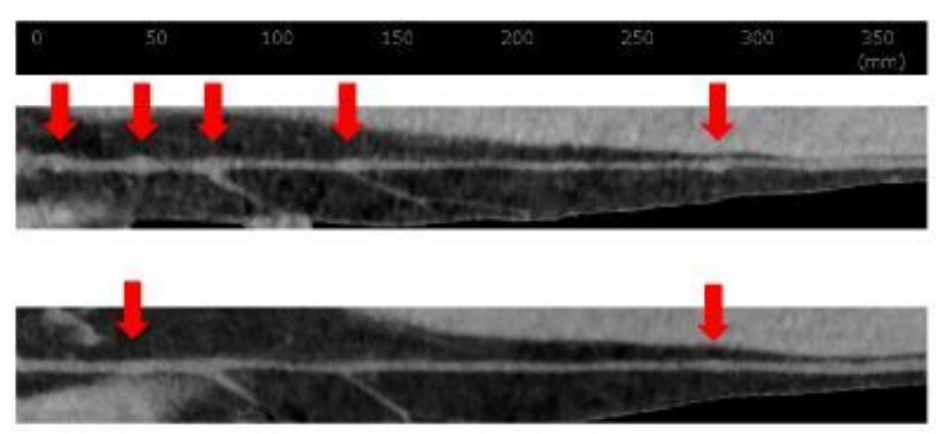

Left

Upright

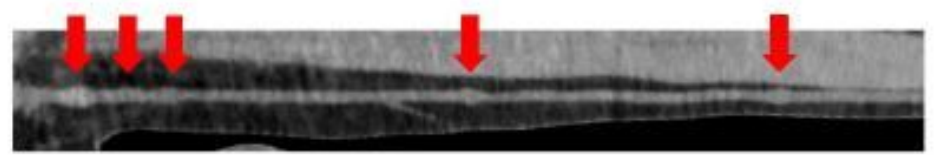

Supine

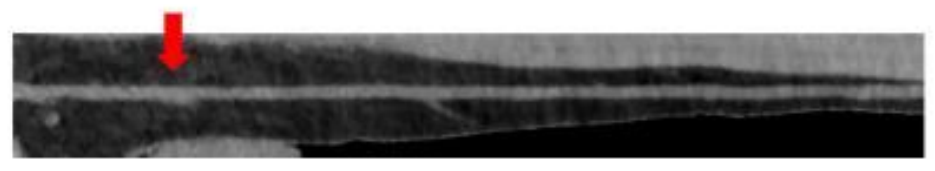

(B)

Right

Upright

Supine

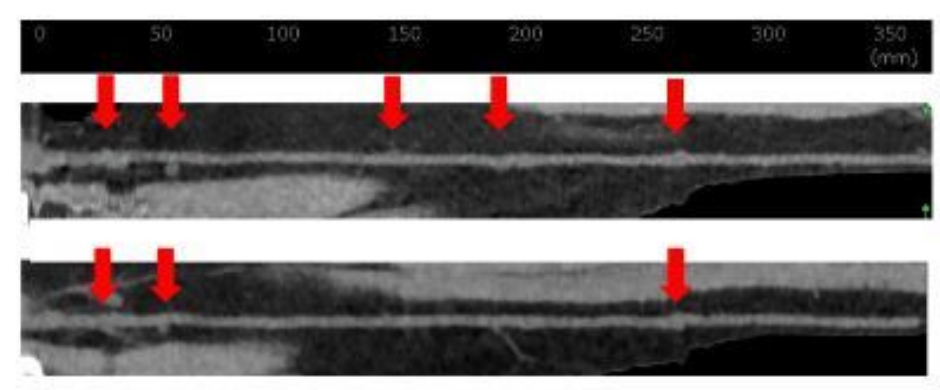

Left

Upright

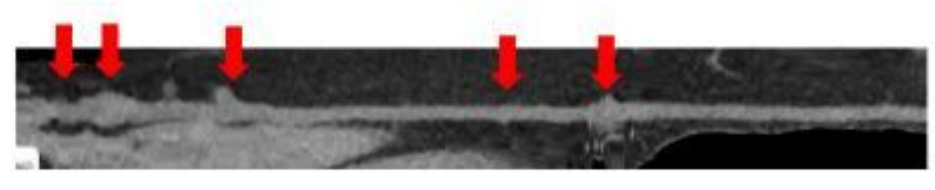

Supine

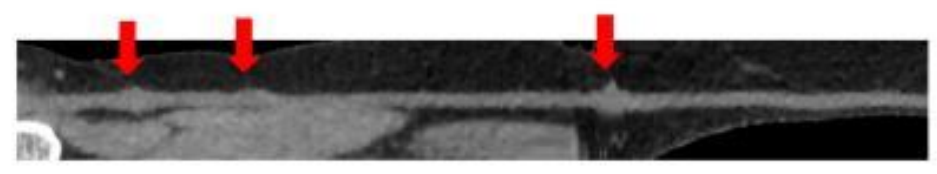

cross

section
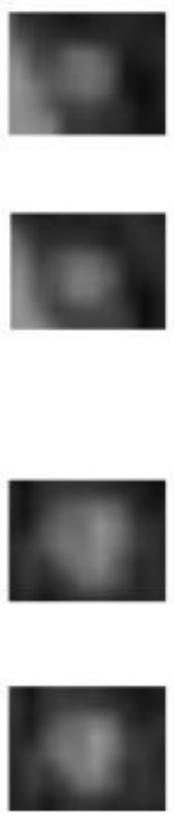

cross

section
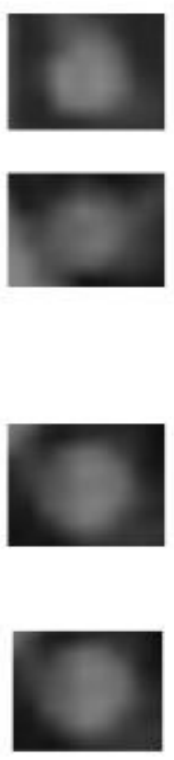

Figure 2 
A represented case from male and female volunteers. (A) A 39-year-old male (B.H. $181.3 \mathrm{~cm}$, B.W. $72.1 \mathrm{~kg}$, BMI 21.9). Upright CT detects $5 / 5$ valves (arrows) and $18 / 13$ tributaries (right/left sides). The crosssection images on the $15 \mathrm{~cm}$ from the SF junction showed the size of 10/22 $\mathrm{mm} 2$ (right/left sides). Supine CT detects $2 / 1$ valves (arrows) and 14/13 tributaries (right/left sides). The cross-section images on the $15 \mathrm{~cm}$ from the $S F$ junction showed the size of $10 / 15 \mathrm{~mm} 2$ (right/left sides). (B) A 52-year-old female (B.H. $162.0 \mathrm{~cm}$, B.W. $47.8 \mathrm{~kg}, \mathrm{BMI} 18.2$ ). Upright CT detects $5 / 5$ valves (arrows) and $14 / 13$ tributaries (right/left sides). The cross-section images on the $15 \mathrm{~cm}$ from the $S F$ junction showed the size of $19 / 29 \mathrm{~mm} 2$ (right/left sides). Supine CT detects $3 / 3$ valves (arrows) and 13/14 tributaries (right/left sides). The cross-section images on the $15 \mathrm{~cm}$ from the SF junction showed the size of $16 / 26 \mathrm{~mm} 2$ (right/left sides). 

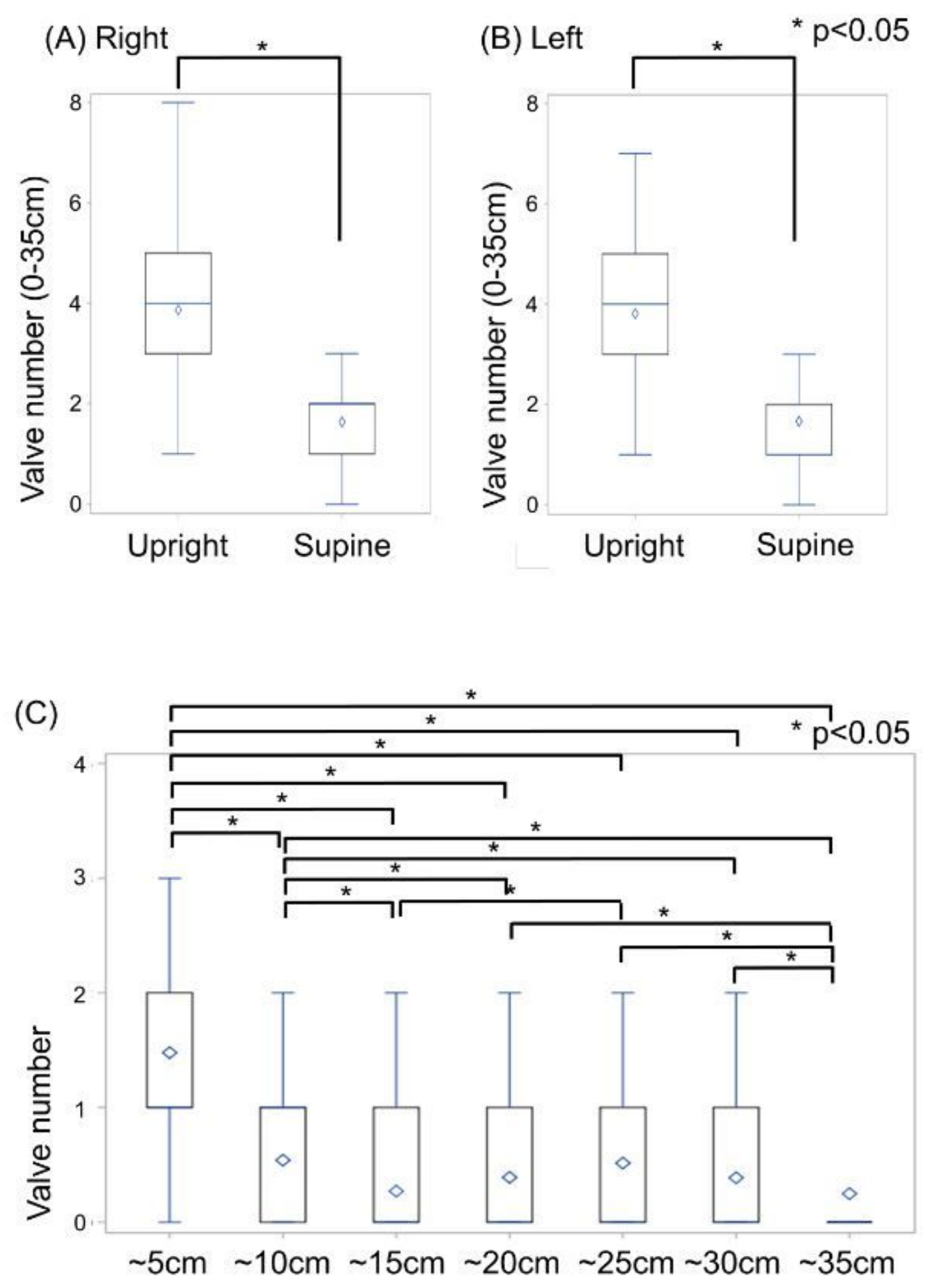

\section{Figure 3}

Valve numbers and distribution in upright and supine CT. The valve numbers in upright and supine $\mathrm{CT}$ in (A) right side and (B) left side. In both sides, the valve numbers are significantly higher in the upright CT. (C) The distribution of valves in upright CT was highest in the lesion from the SF junction to $5 \mathrm{~cm}$, followed by 5 to $10 \mathrm{~cm}$. 
(A)

\section{Right}

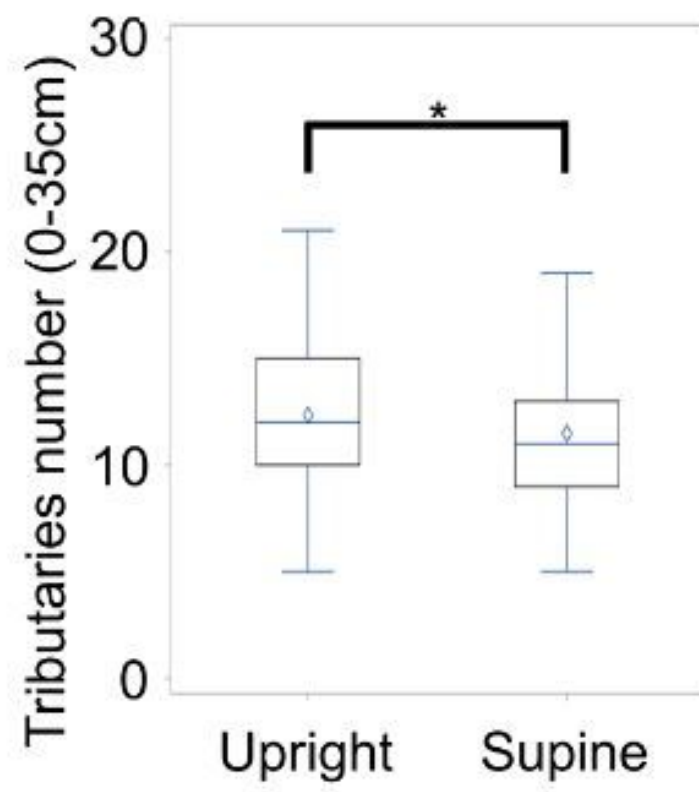

(B)

\section{Right}

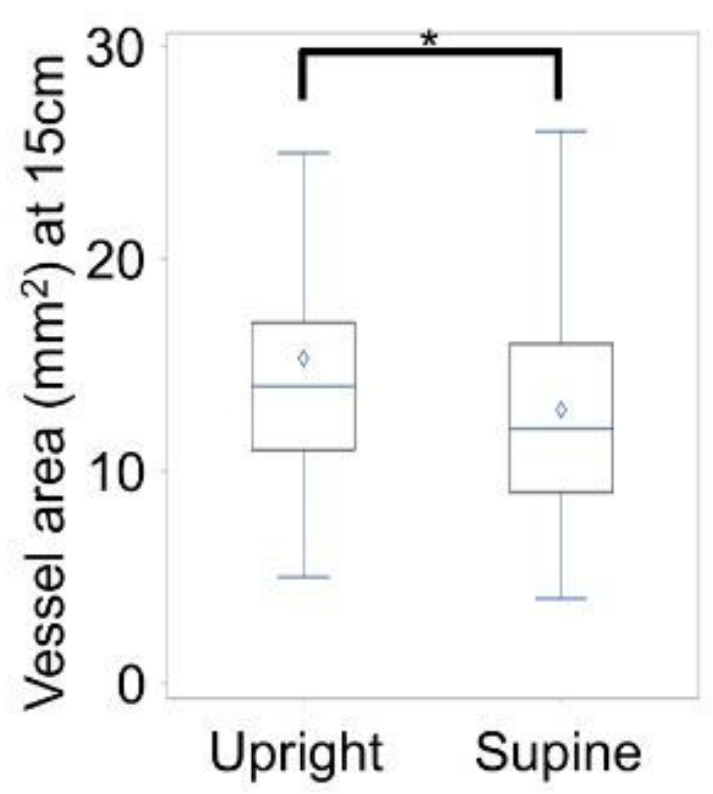

${ }^{*} p<0.05$

\section{Left}

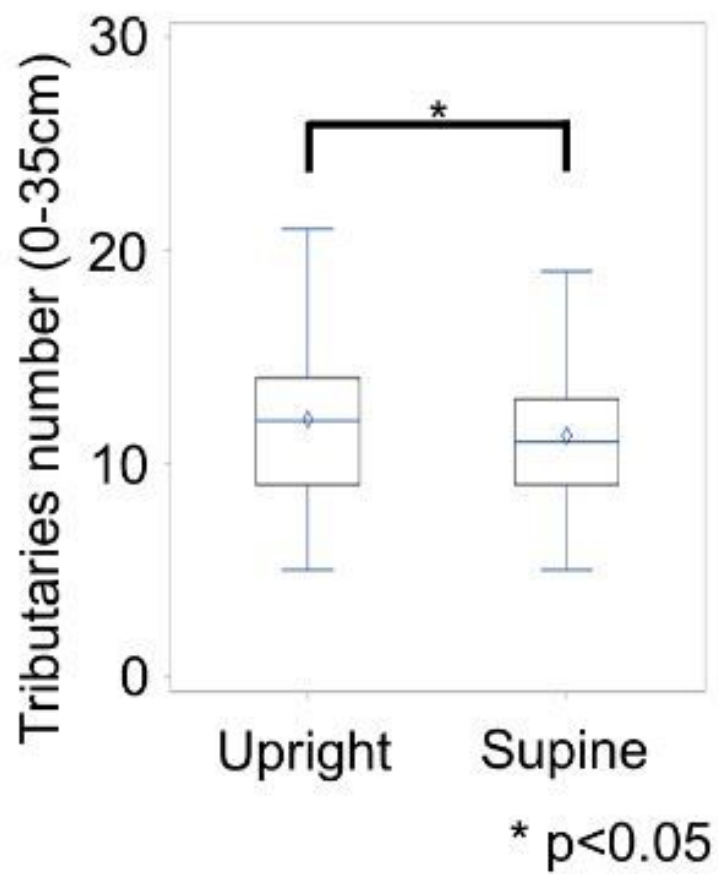

Left

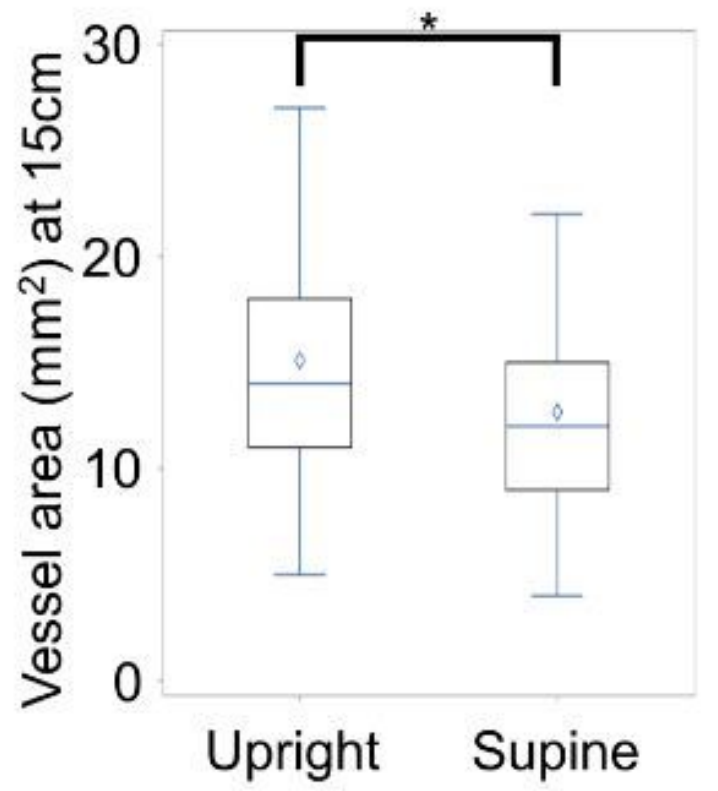

Figure 4

The tributaries numbers and vessel area in upright and supine CT. (A) The tributaries numbers in upright and supine $C T$ in right side and left side. In both sides, the tributaries numbers are significantly higher in the upright CT. (B) The vessel area (15cm from the SF junction) in upright and supine CT in right side and left side. In both sides, the vessel area was significantly higher in the upright CT. 


\section{Supplementary Files}

This is a list of supplementary files associated with this preprint. Click to download.

- SuppleUprightCTSVforScientificReportsver1.0.pdf

- Table1.jpg 